\title{
The Application of Three-Dimensional Collagen-Scaffolds Seeded with Myoblasts to Repair Skeletal Muscle Defects
}

\author{
Jianqun Ma, ${ }^{1,2}$ Kyle Holden, ${ }^{1,3}$ Jinhong Zhu, ${ }^{3}$ Haiying Pan, ${ }^{1,3}$ and Yong $\mathrm{Li}^{4}$ \\ ${ }^{1}$ The Laboratory of Molecular Pathology, Stem Cell Research Center, Children's Hospital of Pittsburgh, PA 15219, USA \\ ${ }^{2}$ Department of Bioengineering, University of Pittsburgh, PA 15219, USA \\ ${ }^{3}$ Department of Orthopedic Surgery, School of Medicine, University of Pittsburgh, PA 15213, USA \\ ${ }^{4}$ Department of Pediatric Surgery, University of Texas, Medical School at Houston, TX 77030, USA \\ Correspondence should be addressed to Yong Li, yong.li.1@uth.tmc.edu
}

Received 30 June 2011; Accepted 11 September 2011

Academic Editor: Guy Benian

Copyright ( $) 2011$ Jianqun Ma et al. This is an open access article distributed under the Creative Commons Attribution License, which permits unrestricted use, distribution, and reproduction in any medium, provided the original work is properly cited.

Three-dimensional (3D) engineered tissue constructs are a novel and promising approach to tissue repair and regeneration. 3D tissue constructs have the ability to restore form and function to damaged soft tissue unlike previous methods, such as plastic surgery, which are able to restore only form, leaving the function of the soft tissue often compromised. In this study, we seeded murine myoblasts (C2C12) into a collagen composite scaffold and cultured the scaffold in a roller bottle cell culture system in order to create a $3 \mathrm{D}$ tissue graft in vitro. The $3 \mathrm{D}$ graft created in vitro was then utilized to investigate muscle tissue repair in vivo. The 3D muscle grafts were implanted into defect sites created in the skeletal muscles in mice. We detected that the scaffolds degraded slowly over time, and muscle healing was improved which was shown by an increased quantity of innervated and vascularized regenerated muscle fibers. Our results suggest that the collagen composite scaffold seeded with myoblasts can create a 3D muscle graft in vitro that can be employed for defect muscle tissue repair in vivo.

\section{Introduction}

Tissue loss usually results in significant dysfunction, physical deformities, and emotional pain long after treatment. Common causes of tissue loss include military injuries, such as high-velocity missiles, fragmenting exploding devices, and penetrating wounds associated with gunshots, and civilian injuries, such as blunt trauma attributed to automobile accidents [1]. Other causes of soft tissue removal include tumor removal, diabetic tissue damage, and irradiative injuries [2-5]. Current treatments of soft tissue injuries, such as skeletal muscle defects, resort to reconstructive plastic surgery, and skin grafting which are effective techniques to be employed in emergency repair and cosmetic applications, but often these techniques are unable to provide restoration of the function to the damaged tissue $[6,7]$.

Recent advances in biotechnology and bioengineering have offered novel approaches to repairing soft tissue defects by combining cell biology, gene transfer, biomaterials, and bioreactors [8-12]. Among the new advances, three-dimensional biodegradable scaffolds provide the ability to restore form and also function to the injured area. In order to be successful, the 3D biodegradable scaffold needs to serve two purposes. First, the scaffold needs to have the physiologic and mechanical properties that mimic the in vivo native environment, and second, the scaffold needs to have the ability to allow for development and remodeling of the tissue in order to promote successful restoration of physiological function [13-15]. It is important for the scaffold to be biodegradable because nondegradable constructs implanted for prolonged time periods often induce an inflammatory response which can often compromise the tissue construct and the healing of the tissue [16-18].

Tissue and organ function are dependent on the presence of appropriate populations of differentiated cells, and therefore, it is important to incorporate proper cells in the 3D scaffold. This can be accomplished through rolling bottle cell culture systems which are advantageous, in comparison to 
static cell seeding, in that a rolling bottle cell culture system improves cell seeding density and homogeneity within the scaffold $[10,19,20]$. This is accomplished through the slow revolutions of the culture bottles on the rolling device which produce a force significant enough to permit the cells to grow evenly in three dimensions [21]. Also the revolutions allow for nutrients to be provided to the cells while at the same time removing waste products through the constant movement of media bathing the cells [22].

In general, the artificial 3D scaffold provides an architecture on which seeded cells can organize and develop into the desired tissue for implantation. Once implanted in the soft tissue defect, the biodegradable scaffold provides an initial biomechanical structure for the replacement tissue until the cells produce an adequate extracellular matrix. During the deposition, organization, and formation of the newly generated extracellular matrix, the scaffold is either degraded or metabolized; eventually leaving a vital organ or tissue that restores, maintains, or improves tissue function. In this study, we built a 3D muscle graft by seeding $\mathrm{C} 2 \mathrm{C} 12$ myoblasts into a biodegradable $3 \mathrm{D}$ collagen composite scaffold, and implanted it into a skeletal muscle defect mouse model in vivo. Immunostaining was employed to evaluate the effectiveness of the graft in improving vascularization, innervation, and regeneration of the damaged tissue.

\section{Materials and Methods}

2.1. C2C12 Myoblasts Transfected with Retroviral LacZ and LacZ Staining. C2C12 myoblasts were purchased from ATCC (American Type Culture Collection) and cultured in media (DMEM, Invitrogen) supplemented with 10\% fetal bovine serum (FBS, Gibco, BR), 10\% horse serum (HS), $1 \%$ penicillin/streptomycin $(\mathrm{P} / \mathrm{S})$, and $0.5 \%$ chick embryo extract (CEE). The culture media was removed and replaced with a $1: 1$ dilution of retroviral LacZ and culture media supplemented with $8 \mu \mathrm{g} / \mathrm{mL}$ of polybrene. The cells were incubated in the solution for 6 hours at $5 \% \mathrm{CO}_{2}$ and $37^{\circ} \mathrm{C}$. To ensure maximum transduction of the virus, the infection was done up to 3 times, and a LacZ staining was done after each infection to determine the efficiency of the infections. The LacZ staining consisted of fixing the cells in $1 \%$ glutaraldehyde solution (Sigma) for 2 minutes followed by washing with PBS. The cells were then incubated for 2 hours in a LacZ solution $(225 \mathrm{~mL}$ PBS $/ 25 \mathrm{~mL} \mathrm{KFE}$ solution $/ 250 \mu \mathrm{L}$ of $2 \mathrm{M} \mathrm{MgCl}_{2} / 5 \mathrm{~mL}$ of X-gal) at $37^{\circ} \mathrm{C}$. The cells were then visualized for analysis using bright field microscopy.

2.2. 3D Collagen Composite Scaffold and Cell Seeding. The 3D collagen composite scaffolds were purchased from BD Biosciences, (catalogue number 354613) and the scaffolds are of a cylindrical shape approximately $4.2-5.2 \mathrm{~mm}$ in diameter and $3.9-4.5 \mathrm{~mm}$ in height with a volume of $0.039 \mathrm{~cm}^{3}$. The average pore size of the scaffold is $100-200 \mu \mathrm{m}$ and the hydration capacity is $25 \mu \mathrm{L}$. The weight and wet weight of the scaffolds are $3.5 \mathrm{mg}$ and $45.0 \mathrm{mg}$, respectively, with an average wet tear strength of $0.48 \pm 0.0131 \mathrm{lb} / \mathrm{mm}$. The collagen composite scaffolds, derived from bovine hide, resemble the structure of collagen within the extracellular matrix, and are able to support both short- and long-term differentiation of multiple cell types. The 3D scaffolds were individually placed into separate wells of a 48 -well plate, and were immersed in a solution consisting of $200 \mu \mathrm{L}$ of culture medium containing approximately 50,000 LacZ ${ }^{+}$myoblasts. The scaffolds were then incubated in $37^{\circ} \mathrm{C}$ at $5 \% \mathrm{CO}_{2}$ for 1 hour.

2.3. Rolling Bottle Cell Culture System. The LacZ ${ }^{+}$myoblast seeded scaffolds were transferred from the 48 -well plates into Corning cell culture bottles containing $200 \mathrm{~mL}$ of culture media. The Corning cell culture bottles were placed on a Wheaton rolling device (Fischer Scientific) at a speed of 5 revolutions per minute (RPM). The Wheaton rolling device was then placed in an incubator at $37^{\circ} \mathrm{C}$ and $5 \% \mathrm{CO}_{2}$ for 3 weeks with periodic cell culture media changes.

2.4. Scaffold Sectioning and Immunostaining. In vitro, the scaffolds were harvested after 1,2, and 3 weeks of culturing, and rinsed with PBS before being snap-frozen in 2-methyl butane precooled in liquid nitrogen and stored at $-80^{\circ} \mathrm{C}$. The scaffolds were then cryosectioned at $10 \mu \mathrm{m}$ and prepared for immunostaining.

In vivo, scaffolds were harvested at various time points, and immunostaining was performed to detect CD31, vWF, dystrophin, neurofilament protein, and fast MyHC expression in injured GMs implanted with the myoblast seeded scaffolds. Tissue sections were fixed in $4 \%$ formalin for 5 minutes followed by washing with PBS. Nonspecific binding was blocked with $10 \% \mathrm{HS}$ for 1 hour at room temperature (RT). Primary antibodies were diluted to $1: 200$ in $2 \%$ HS and incubated overnight at $4^{\circ} \mathrm{C}$. The sections were then rinsed with PBS followed by secondary antibody incubation for 1 hour at RT in a $1: 300$ dilution. The sections were then rinsed with PBS before the nuclei were counterstained with DAPI.

2.5. Proliferation Assay. After 10 days of culturing the scaffolds in the Wheaton rolling device, a BrdU assay was performed in order to evaluate the proliferation capacity of the $\mathrm{C} 2 \mathrm{C} 12$ myoblasts within the scaffold. BrdU was added to growth medium at dilution of $1: 1000$ for an incubation period of 24 hours. The scaffolds were then removed from the medium and rinsed with PBS before being snap-frozen in 2 -methyl butane precooled in liquid nitrogen. The scaffolds were then sectioned and fixed with $4 \%$ formalin. The DNA was denatured for $10 \mathrm{~min}$ on ice with $1 \mathrm{~N} \mathrm{HCl}$ followed by $2 \mathrm{~N} \mathrm{HCl}$ at RT for $10 \mathrm{~min}$, and finally $2 \mathrm{~N} \mathrm{HCl}$ for $20 \mathrm{~min}$ at $37^{\circ} \mathrm{C}$. Nonspecific binding was blocked with $10 \%$ HS for 1 hour. The primary antibody (biotin conjugated anti-BrdU) was added at a 1:250 dilution in 2\% HS for 3 hours at RT. The secondary antibody (biotin streptavidin) was then added at a dilution of $1: 400$ for 1 hour at RT. The nuclei were then counterstained with DAPI.

2.6. Animal Experiments. The animal protocol was approved by the Animal Research and Care Committee at Children's Hospital of Pittsburgh. The mice included normal (C57BL6J, 5-8 weeks of age, male), SCID (C57BL/6J-Prkdc ${ }_{\text {scid }} /$ SzJ, 48 weeks of age, female), and dystrophic/immunodeficient 
$m d x / S C I D\left(C 57 B L / 10 S c S n-D m d_{m d x}\right.$ crossed with C57BL/6J$\operatorname{Prkdc}_{\text {scid }} /$ SzJ, 4-8 weeks of age, female). All mice were purchased from Jackson Laboratory (Bar Harbor, Maine), and were housed individually with proper access to food and water and maintained on a 12-hour light/dark cycle. After the mice were anesthetized, an approximately $4 \times 4 \times 3 \mathrm{~mm}^{3}$ defect was created in the belly of the gastrocnemius muscle (GM). The 3D muscle grafts and control nonseeded scaffolds were implanted in the defects followed by immediate wound closure. At designed time periods, mice were sacrificed, and the GMs were harvested and snap-frozen in 2-methylbutane precooled in liquid nitrogen, and they were cryosectioned for histological analysis.

\section{Results}

3.1. In Vitro. In the in vitro portion of the experiments, the C2C12 myoblast seeded scaffolds were cultured in a roller bottle cell culture system, and the scaffolds were harvested after 1, 2, and 3 weeks of culturing. After 1 week of culture, myoblasts were distributed throughout the interior of the scaffold which provides evidence that extensive migration was able to occur (Figures 1(a)-1(f)). Some of the cells migrated into the interior region of the scaffold, and this can be detected by either HE (Figures $1(\mathrm{a})-1(\mathrm{c})$ ) or LacZ (Figures 1(d)-1(f)) staining. The sectioned scaffolds were also immunostained for CD31, an endothelial marker, and vWF, an endothelial cell-specific protein (Figures 2(a)-2(c)). After 1 week of culture, CD31positive cells were seen within the scaffold (red, Figure 2(a)). Colocalization between CD31positive (red) and vWF-positive (green) cells was visualized after 2 weeks of culture (yellow, Figure 2(b)). The detection of the colocalization of CD31 positive and vWF positive cells is indicative of vascular development within the scaffold. After 3 weeks of culturing, more extensive colocalized positive cells were seen (yellow, Figure 2(c)). A BrdU assay was performed in order to assess the viability and proliferative capacity of the myoblasts within the scaffold. We detected myoblasts with nuclei positive for BrdU (red, Figures 2(d), 2(e), and $2(\mathrm{f})$ ), revealing that some of the myoblasts seeded within the scaffold were able to proliferate.

3.2. In Vivo. In the normal mouse model, implantation of a nonseeded control scaffold resulted in rejection from the host tissue, and this was seen 20 days after implantation (Figure 3(a)). In the SCID mouse model, the implanted nonseeded scaffolds were not rejected by the host tissue, but few host cells migrated into the area of the implantation (Figure 3(b)). In the same SCID mouse model, implantation of the 3D muscle graft with LacZ-positive myoblasts for a duration of 20 days, resulted in detection of large populations of LacZ-positive cells remaining in the transplantation site as well as extensive migration of the LacZ-positive cells into the surrounding host tissue, which demonstrates the success of the 3D graft implantation into the host tissue (Figure 3(c)). With immunostaining, we discovered CD31 and vWF positive cells present within the interior of the scaffold, and some of these cells expressed colocalization of the two markers

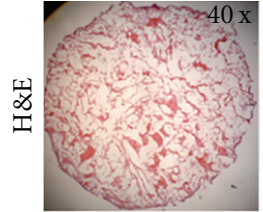

(a)

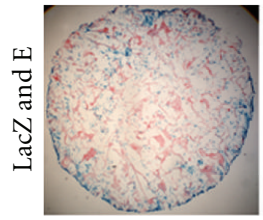

(d)

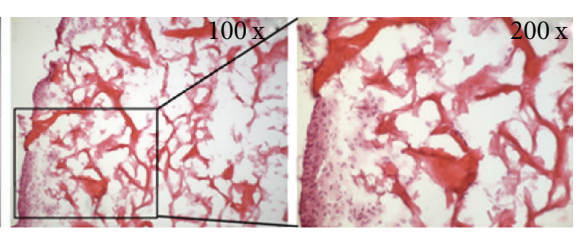

(b) (c)

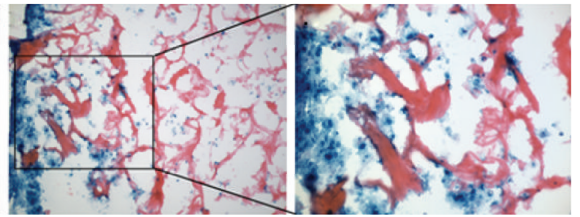

(e)

(f)
Figure 1: Cell growth examined after 1 week of culturing in vitro. Cells were visualized throughout the scaffold with a higher concentration of cells located on the periphery of the scaffolds; however, extensive migration is seen within the scaffolds. H\&E staining of the scaffolds ((a)-(c)), and LacZ and eosin staining ((d)(f)) are shown.

(Figure $3(\mathrm{~d})$ ), indicating that the vasculature process had began to develop.

In the $m d x / S C I D$ mouse model, we followed the same procedure described above for the normal mouse model. We observed that the trauma sites were quickly healed in the 3D muscle graft implanted skeletal muscle after 10 (Figure 4(a)) and 20 days (Figure 4(c)) after surgery in comparison to the healing seen in the nonseeded scaffolds implanted into the muscle (Figures 4(b) and 4(d)). By histological analysis, the LacZ-positive cells were able to survive within the implanted scaffold as well as migrate throughout the scaffold. The scaffold began to breakdown as it was incorporated within the surrounding host tissue 10 days after implantation (Figures 4(e) and 4(f)). Twenty days after the graft implantation, we also detected that multiple cell types were spread homogeneously throughout the site of injury, and significantly, we detected CD31 and vWF positive cells present in the new tissue and some of these cells demonstrated colocalization between these two markers (Figures $4(\mathrm{~g})$ and $4(\mathrm{~h})$ ). This result indicates that the vascularization process had started within the $3 \mathrm{D}$ muscle graft as early as 10 days after implantation.

We also investigated the histology of the long-term 3D muscle graft after implantation in the $m d x /$ SCID mouse model. One month after implantation of the 3D muscle graft, LacZ and eosin staining provided evidence that the LacZ-positive myoblasts were able to survive within the implanted scaffold (Figure 5(a)). The site of injury was composed of LacZ-positive myoblasts and nondegraded residues of the scaffold (Figure 5(a) and 5(c) arrows). Some LacZ-positive myoblasts were detected at the periphery of the scaffold forming LacZ positive multinucleated myotubes (Figure 5(a), arrowheads). Many desmin positive cells (arrowheads), indicative of mature muscle cells, were detected within the area of implantation around nondegraded regions of the scaffold (Figure 5(b), arrows). With an H\&E staining, we detected newly formed myofibers within the region of the degrading scaffold (Figure 5(d)). 


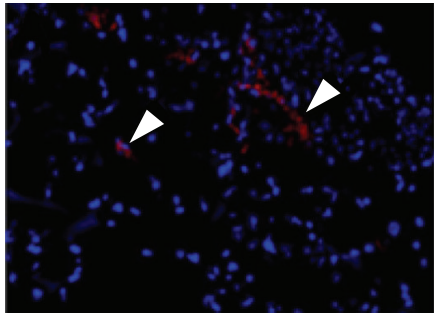

(a)

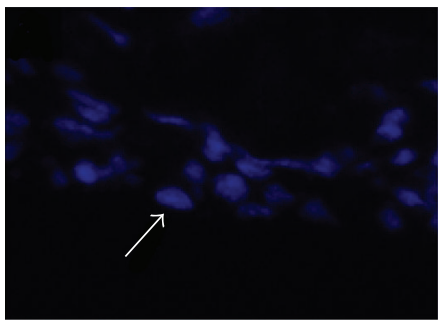

(d)

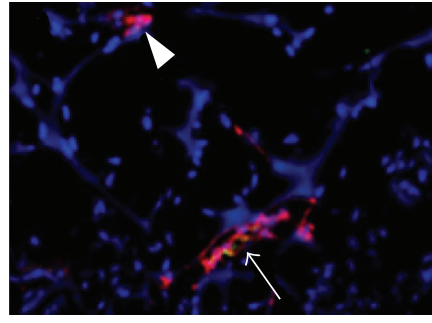

(b)

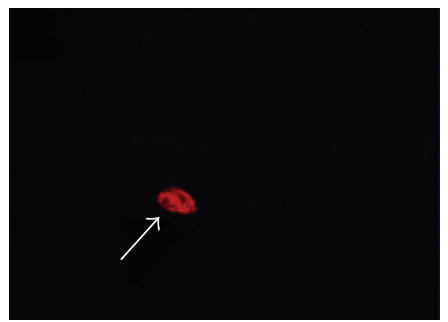

(e)

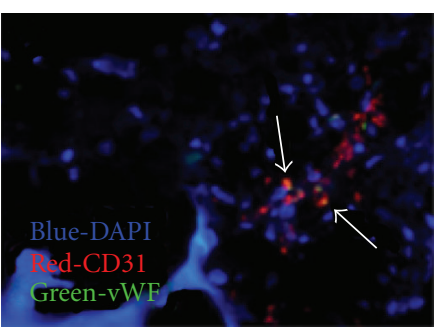

(c)

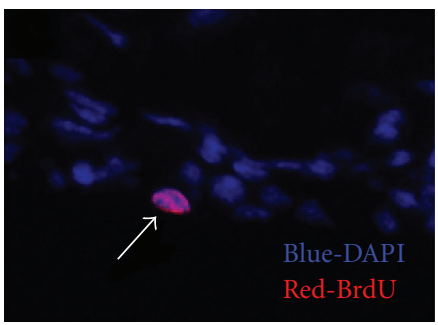

(f)

Figure 2: Immunofluorescent staining of the scaffolds after culturing for 1, 2, and 3 weeks in vitro for evidence of vasculature ((a)-(c)). After one week of culturing (a), CD31 positive cells ( red) are detected within the scaffold. After two weeks of culturing (b), colocalization of CD31 (red) and vWF (green) positive cells are visualized. After three weeks of culturing (c), more extensive colocalization of CD31 and vWF is seen. A BrdU assay was performed in vitro after 10 days of culturing ((d)-(f)). BrdU positive nuclei (red) were seen revealing that proliferation was occurring throughout the scaffold (e).

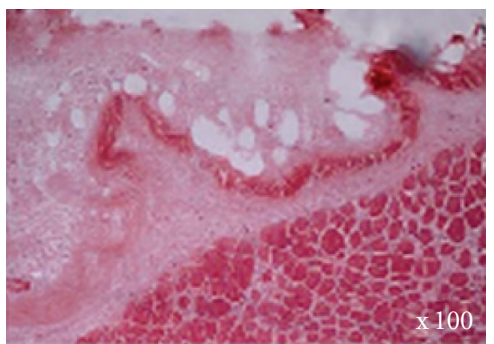

(a)

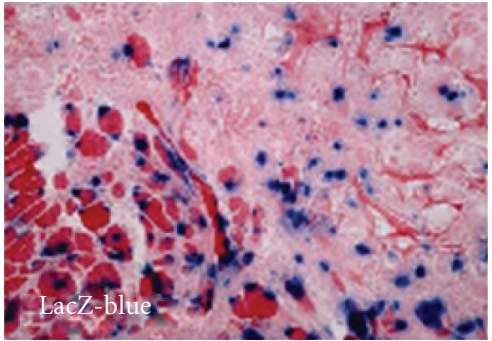

(c)

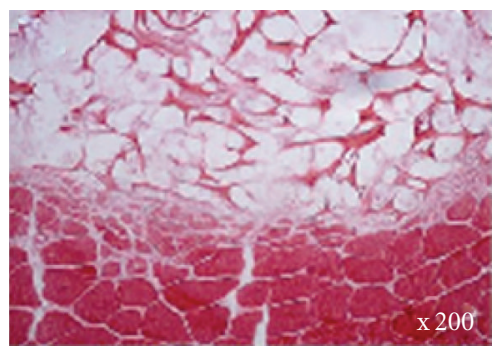

(b)

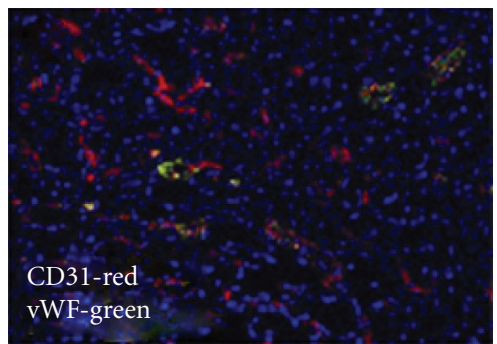

(d)

FIGURE 3: The immunocompatibility of the scaffolds was examined in normal mice and SCID mice in vivo. Twenty days after implantation in the normal mouse model, nonseeded scaffolds were rejected by the host tissue (a). Twenty days after implantation in the SCID mouse model, nonseeded scaffolds were not rejected by the host tissue, and few host cells migrated into the scaffold (b). Twenty days after implantation in the SCID mouse model, LacZ-labeled C2C12 myoblast seeded scaffolds were well received in the host tissue (c). Also, LacZ ${ }^{+}$cells were able to migrate into the surrounding host tissue. An immunofluorescent staining of CD31 (red) and vWF (green) was performed on the implanted seeded scaffolds 20 days after implantation $(\mathrm{d})$.

With immunostaining, dystrophin-positive myofibers were detected within the implantation region which suggests that regeneration was occurring within the site of injury (Figure 5(e), arrowheads). We also discovered that some CD31 positive cells were located within the site of injury, revealing that the vascularization was an ongoing process (Figures 5(e) and 5(f), arrows).

Two months after implantation of the 3D muscle grafts, large dystrophin positive grafts were detected within the site of injury suggesting that muscle regeneration had taken 

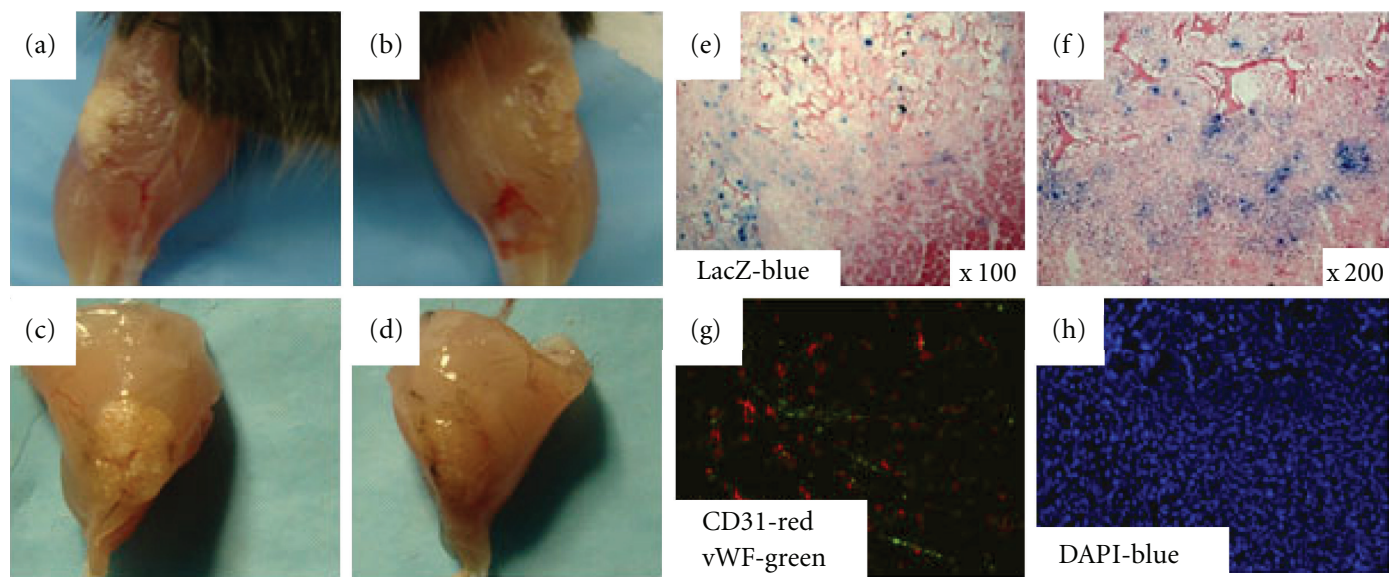

FIgURE 4: In the $m d x /$ SCID mouse model, healthy healing was observed in the myoblast seeded scaffolds after 10 days (a) and 20 days (c). Nonseeded scaffolds implanted into $m d x /$ SCID mice resulted in slower and less healthy healing at 10 days (b) and 20 days (d). LacZ staining of the implanted muscle graft region revealed extensive migration of the LacZ ${ }^{+}$myoblasts into the surrounding host tissue (e) and (f). Immunofluorescent staining of CD31 (red) and vWF (green) showed vascularization developing in the implanted muscle graft region 20 days after implantation (g). A DAPI staining revealed that a variety of cell types were located homogenously throughout the implanted muscle graft area $(\mathrm{h})$.
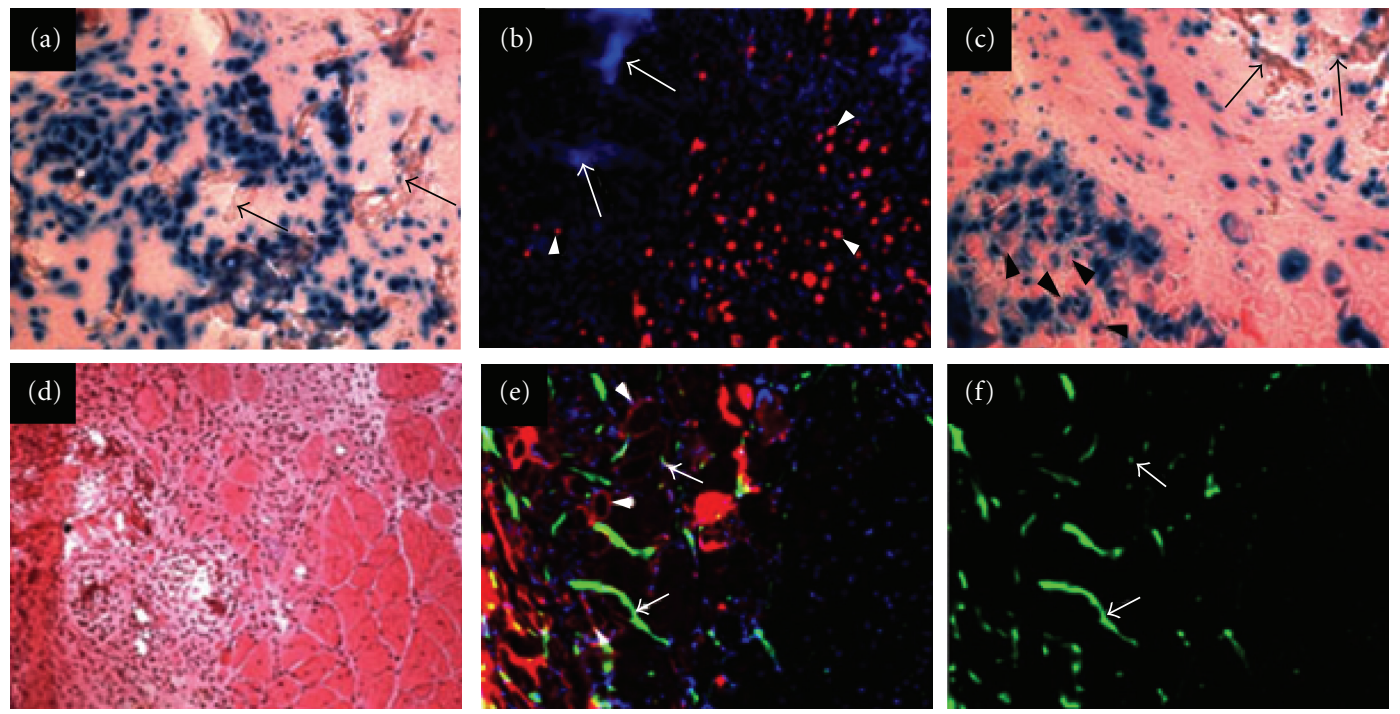

FIGURE 5: LacZ and eosin staining 1 month after implantation revealed that numerous LacZ ${ }^{+}$myoblasts were able to survive within the implanted muscle graft region ((a) and (c)). Residues of nondegraded scaffold were also visualized within the implanted muscle graft area ((c), arrowheads). LacZ ${ }^{+}$myoblasts were seen forming multinucleated myotubes within the implanted area ((a) arrows). Desmin positive myoblasts ((b) arrowheads) were detected within the vicinity of nondegraded portions of the scaffold ((b) arrows). An H\&E staining revealed newly formed myofibers within the implantation site (d). Dystrophin-positive myofibers (red, arrowheads) were detected 1 month after implantation (e) as well as CD31-positive cells, ((e) and (f), green).

place in many regions of the injured area (Figures 6(a) B, $\mathrm{E}$, and $\mathrm{H}$ ). Also, the majority of the dystrophin-positive grafts stained positive for fast myosin-heavy chain protein (Figures 6(a) G-I). Similar to the previous time points, CD31 was visualized which provides evidence for extensive vascular development within the site of injury (Figures 6(a) A-C). Also neurofilament protein was detected within the dystrophin-positive grafts suggesting that innervations were being formed to the regenerating myofibers (Figure 6(a)
D-F). With histological analysis, we have detected a large area of scar tissue remaining in the implanted 3D graft sites three months after implantation through an H\&E staining (Figure 6(b) A and B, stars). Also, we have detected regeneration ofmyofibers (Figure 6(b) B, arrows) and large amounts of dystrophin-positive myofibers within the new muscle graft (Figure 6(b) C and D, dystrophin-red, arrows) with revascularization (Figure 6(b) D, CD31-green, arrowheads). This existing scar tissue may be a major factor to 


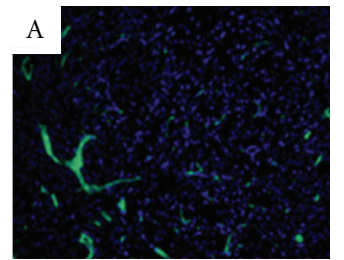

CD31

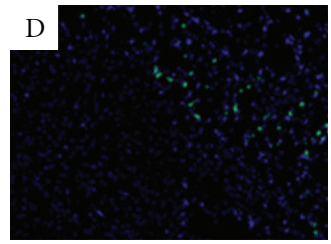

Neurofilament

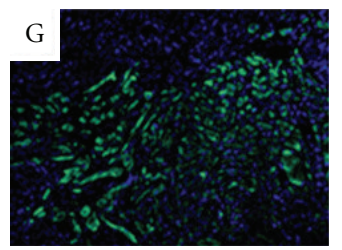

$\mathrm{MyHC}$

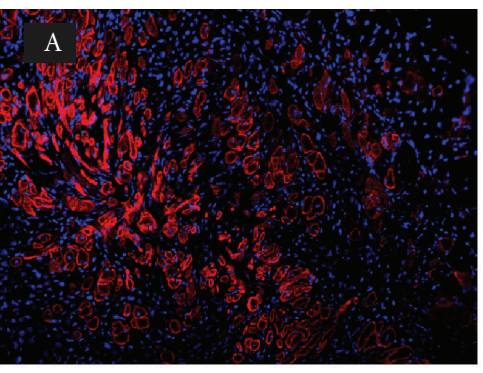

Dystrophin

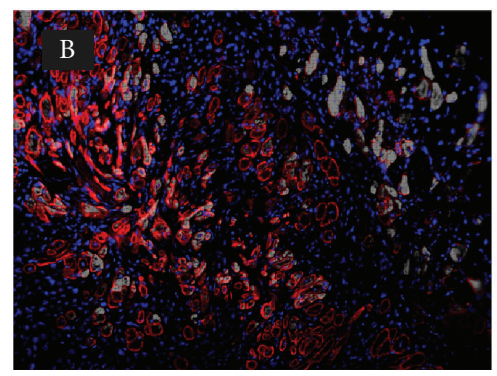

Dystrophin + slow MyHC

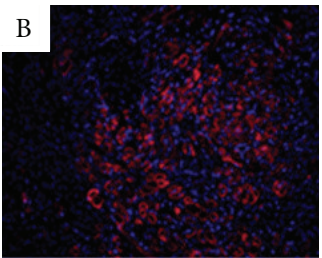

Dystrophin

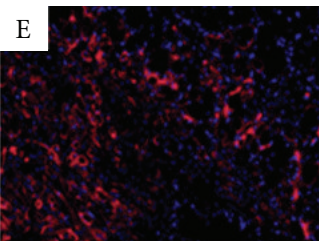

Dystrophin

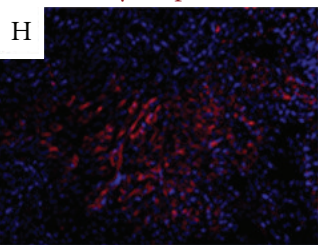

Dystrophin

(a)

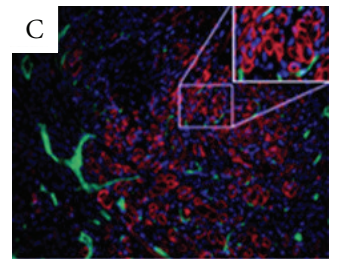

Merged

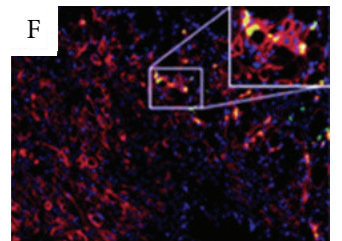

Merged

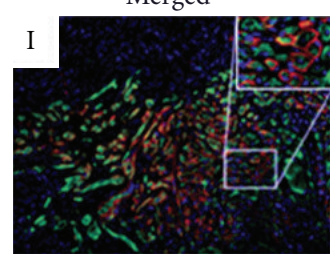

Merged

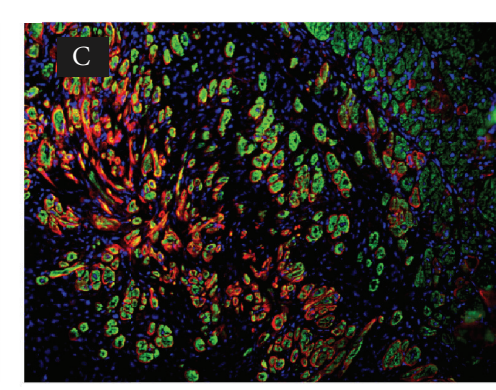

Dystrophin + fast MyHC

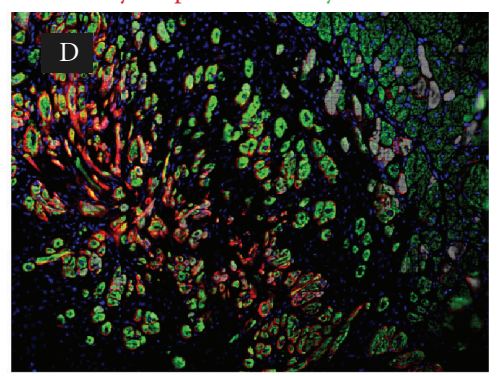

Dys + slow MyHC + fast MyHC

(b)

Figure 6: (a) Three months after implantation in vivo, large dystrophin-positive grafts were visualized ((a) B, E, H). CD31-positive cells were detected within the dystrophin-positive grafts indicative that vascularization and regeneration were occurring ((a) A-C). Neurofilament protein was visualized within the dystrophin-positive grafts revealing that innervations were forming along with the regenerating myofibers ((a) D-F). The majority of the dystrophin-positive grafts stained positively for fast myosin-heavy chain protein ((a) G-I). (b) Three months after implantation in vivo, all newly formed myofibers stained positive for dystrophin ((b) A dystrophin-red). A small quantity of the dystrophin-positive myofibers stained positive for slow myosin-heavy chain protein ((b) B slow MyHC-gray). A larger quantity of the dystrophin-positive myofibers stained positive for fast myosin-heavy chain protein ((b) C fast MyHC-green). (b) D displays a merged image revealing the distribution of the two types of myosin-heavy chain proteins. 

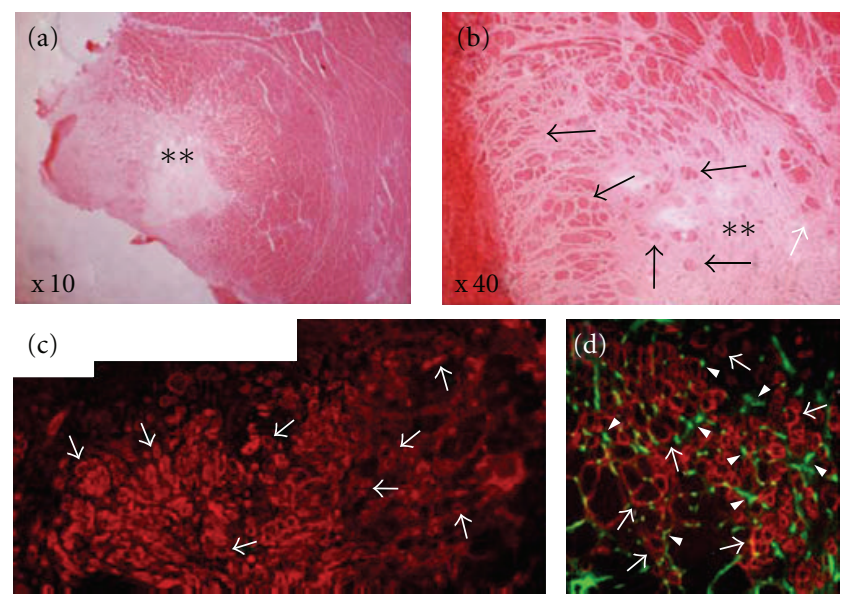

Figure 7: Three months after implantation, evaluation of the regenerative capacity of the seeded scaffolds. A hematoxylin and eosin staining revealed a large area of fibrosis in the muscle graft ((a) and (b), sk). However, within the muscle graft despite the area of fibrosis, we detected regenerating myofibers ((b), arrows $\mathrm{f}$ ), and large quantities of dystrophin-positive myofibers ((c), arrows f). Within the areas of regeneration, revascularization was detected through the visualization of CD31-positive cells ((d) green/arrows f).

interfere with further muscle functional recovery, and can be a potential inducer of muscle reinjury with movement. Thus, prevention of fibrosis is necessary for the success of the bioengineered tissue in application.

\section{Discussion}

In the last few decades, advances in bioengineering have moved the field of tissue engineering significantly forward $[15,23,24]$. While 3D tissue constructs are still being developed, this field continues to grow from the intense efforts of military and civilian trauma researchers. Tissue engineering techniques combined with cell biology and material sciences hold great promise in developing appropriate strategies for the repair and regeneration of biological tissues. The necessary components for successful 3D engineered tissues have been identified to include the following: a biodegradable material that serves as a scaffold to facilitate new tissue growth, and pluripotent or stem cells to initiate new tissue growth as well as promoting vascularization and innervation once implanted within the patient. Our work focused primarily on creating functional $3 \mathrm{D}$ tissue constructs in vitro, through the use of a $3 \mathrm{D}$ biodegradable scaffold seeded with $\mathrm{C} 2 \mathrm{C} 12$ primary myoblasts, which could then be implanted in vivo for the purpose of repairing and replacing damaged and lost tissue due to soft tissue defects.

Our results confirmed that $\mathrm{C} 2 \mathrm{C} 12$ myoblasts are able to survive and migrate within the $3 \mathrm{D}$ collagen composite scaffold in vitro. This is a necessary step to occur prior to implantation in order to ensure that the implanted scaffolds are incorporating a rich source of myoblasts into the area of the skeletal muscle injury. Also, we discovered that in vitro, CD31-positive cells were present after 1 week of culturing.
CD31 is an endothelial marker that signifies progenitor blood vessel cells $[25,26]$ which means that the cell seeded scaffold had already started to develop some form of vasculature prior to implantation. This is furthered by evidence that after 2 weeks of culturing in vitro, colocalization between CD31- and vWF-positive cells was detected. These results indicate that the cell seeded scaffolds are capable of forming a type of prevascularization which is ideal for inducing vascular development within the defected muscle once implanted.

The ability to promote vascularization is an essential aspect of tissue engineering because often the vascularization to the area where the tissue loss took place is compromised by the trauma itself [4]. The vascularization that was seen in vitro was also present when the scaffolds were harvested at various time points after implantation in vivo. The detection of CD31-positive cells located throughout the scaffold provide evidence that vascularization was being incorporated within the scaffold from vessels located in the host tissue surrounding the defect. This incorporation of vasculature within the scaffold increased the cell survival of the myoblasts by providing nutrients and oxygen to the newly transplanted cells $[27,28]$. In order for a tissue construct to be effective in becoming functional, vascularization needs to occur along with innervation. Techniques for repairing soft tissue defects, such as muscle and perforator flaps, result in providing vascularized coverage to the wound; however, due to the lack of innervations to the damaged area, sen sitivity is often compromised [6]. Our results indicated that the seeded scaffold was successful in providing potential innervations to the damaged tissue based on the presence of neurofilament-positive cells. Neurofilament protein is a protein located in the intermediate filament of neurons [29]; therefore, its expression is indicative of neurogenesis and neural development.

Dystrophin-positive myofibers were found located within the area of the defect which signifies that new muscle fibers were being formed in the damaged area. The myoblasts that were seeded in the scaffold were able to successfully differentiate into myotubes which allowed for regeneration of the damaged tissue. This result is not surprising due to numerous previous studies that found that myoblast transplantation is an effective therapy for increasing dystrophinpositive myofibers in such muscle dystrophy diseases such as Duchenne muscular dystrophy [30-32]. A large quantity of the dystrophin-positive myofibers stained positively for fast-type myosin-heavy chain protein, and a lower quantity staining positively for slow-type myosin-heavy chain protein. This preferential difference may be contributed to the environment into which the myoblasts were implanted [33]. If more fast-type myosin-heavy chain myofibers were present in the surrounding area of host tissue around the defect, differentiation of the myoblasts into myotubes exhibiting fasttype myosin-heavy chain proteins may have been favored.

Although many challenges still remain in the successful restoration of soft tissue defects, our study provides a starting point for overcoming these challenges. The ability of the myoblast seeded collagen composite scaffolds to provide the initial architecture for rebuilding a functional tissue, as well as incorporating an appropriate population of cells that helps 
to facilitate muscular regeneration through increasing innervation, vascularization, and the production of myofibers, makes the $3 \mathrm{D}$ collagen scaffold an ideal candidate for repairing soft tissue defects. However, immunocompatibility and fibrosis are two problems that still remain. As seen in our data, only immune-compromised mice were able to successfully integrate the $3 \mathrm{D}$ tissue graft into their host tissue which presents a limited scope of application for the scaffolds. Also, fibrosis is a pathological process that affects many tissues and organs after being afflicted by injuries or disease-like states [34]. The development of the fibrotic tissue can lead to chronic healing problems, which can result in tissue/organ dysfunction during the final healing stages, and can make the tissue more susceptible to reinjury after initial healing $[35,36]$. Our study detected fibrosis formation in the skeletal muscles after long-term implantation, indicating that prevention or treatment of the fibrous scar tissue is essential for effective bioengineered tissues.

\section{Author's Contributions}

J. Ma and K. Holden contributed equally to this paper.

\section{References}

[1] M. Dragas, L. Davidovic, D. Kostic et al., "Upper extremity arterial injuries: factors influencing treatment outcome," Injury, vol. 40, no. 8, pp. 815-819, 2009.

[2] C. E. Attinger, I. Ducic, P. Cooper, and C. M. Zelen, "The role of intrinsic muscle flaps of the foot for bone coverage in foot and ankle defects in diabetic and nondiabetic patients," Plastic and Reconstructive Surgery, vol. 110, no. 4, pp. 10471054, 2002.

[3] A. K. Singh, K. P. Gudehithlu, S. Patri et al., "Impaired integration of endothelial progenitor cells in capillaries of diabetic wounds is reversible with vascular endothelial growth factor infusion," Translational Research, vol. 149, no. 5, pp. 282-291, 2007.

[4] M. M. Al-Qattan, "Severe, traumatic soft-tissue loss in the antecubital fossa and proximal forearm associated with radial and/or median nerve palsy: nerve recovery after coverage with a pedicled latissimus dorsi muscle flap," Annals of Plastic Surgery, vol. 46, no. 2, pp. 125-129, 2001.

[5] A. Takeuchi, H. Tsuchiya, T. Shirai, K. Hayashi, H. Nishida, and K. Tomita, "Occlusive dressing for large soft tissue defects following soft tissue tumor excision," Journal of Orthopaedic Science, vol. 14, no. 4, pp. 385-390, 2009.

[6] E. D. Rodriguez, R. Bluebond-Langner, C. Copeland, T. N. Grim, N. K. Singh, and T. Scalea, "Functional outcomes of posttraumatic lower limb salvage: a pilot study of anterolateral thigh perforator flaps versus muscle flaps," The Journal of trauma, vol. 66, no. 5, pp. 1311-1314, 2009.

[7] X. Shao, C. Chen, X. Zhang, Y. Yu, D. Ren, and L. Lu, "Coverage of fingertip defect using a dorsal island pedicle flap including both dorsal digital nerves," Journal of Hand Surgery, vol. 34, no. 8, pp. 1474-1481, 2009.

[8] P. A. Janmey, J. P. Winer, and J. W. Weisel, "Fibrin gels and their clinical and bioengineering applications," Journal of the Royal Society Interface, vol. 6, no. 30, pp. 1-10, 2009.

[9] S. G. Kumbar, S. P. Nukavarapu, R. James, L. S. Nair, and C. T. Laurencin, "Electrospun poly(lactic acid-co-glycolic acid) scaffolds for skin tissue engineering," Biomaterials, vol. 29, no. 30, pp. 4100-4107, 2008.

[10] M. Pei, L. A. Solchaga, J. Seidel et al., "Bioreactors mediate the effectiveness of tissue engineering scaffolds," The FASEB Journal, vol. 16, no. 12, pp. 1691-1694, 2002.

[11] S. A. Riboldi, M. Sampaolesi, P. Neuenschwander, G. Cossu, and S. Mantero, "Electrospun degradable polyesterurethane membranes: potential scaffolds for skeletal muscle tissue engineering," Biomaterials, vol. 26, no. 22, pp. 4606-4615, 2005.

[12] L. G. Griffith, "Emerging design principles in biomaterials and scaffolds for tissue engineering," Annals of the New York Academy of Sciences, vol. 961, pp. 83-95, 2002.

[13] L. E. Freed, F. Guilak, X. E. Guo et al., "Advanced tools for tissue engineering: scaffolds, bioreactors, and signaling," Tissue Engineering, vol. 12, no. 12, pp. 3285-3305, 2006.

[14] D. W. Hutmacher, "Scaffold design and fabrication technologies for engineering tissues - state of the art and future perspectives," Journal of Biomaterials Science, Polymer Edition, vol. 12, no. 1, pp. 107-124, 2001.

[15] L. Cen, W. Liu, L. Cui, W. Zhang, and Y. Cao, "Collagen tissue engineering: development of novel biomaterials and applications," Pediatric Research, vol. 63, no. 5, pp. 492-496, 2008.

[16] J. M. Anderson and J. J. Langone, "Issues and perspectives on the biocompatibility and immunotoxicity evaluation of implanted controlled release systems," Journal of Controlled Release, vol. 57, no. 2, pp. 107-113, 1999.

[17] J. E. Babensee, J. M. Anderson, L. V. McIntire, and A. G. Mikos, "Host response to tissue engineered devices," Advanced Drug Delivery Reviews, vol. 33, no. 1-2, pp. 111-139, 1998.

[18] J. M. Anderson, "Inflammatory response to implants," ASAIO Transactions, vol. 34, no. 2, pp. 101-107, 1988.

[19] G. Vunjak-Novakovic, B. Obradovic, I. Martin, P. M. Bursac, R. Langer, and L. E. Freed, "Dynamic cell seeding of polymer scaffolds for cartilage tissue engineering," Biotechnology Progress, vol. 14, no. 2, pp. 193-202, 1998.

[20] M. Radisic, M. Euloth, L. Yang, R. Langer, L. E. Freed, and G. Vunjak-Novakovic, "High-density seeding of myocyte cells for cardiac tissue engineering," Biotechnology and Bioengineering, vol. 82, no. 4, pp. 403-414, 2003.

[21] Y. Wang, H. L. Jiao, J. Z. Zhang, and R. Q. He, "Threedimensional culture of hybridoma cells secreting anti-human chorionic gonadotropin by a new rolling culture system," Journal of Biomedicine and Biotechnology, vol. 2004, no. 1, pp. 35-40, 2004.

[22] N. A. Bleckwenn and J. Shiloach, "Large-scale cell culture," Current Protocols in Immunology, Appendix 1, Appendix 1U, 2004.

[23] D. H. Park, C. V. Borlongan, D. J. Eve, and P. R. Sanberg, "The emerging field of cell and tissue engineering," Medical Science Monitor, vol. 14, no. 11, pp. RA206-RA220, 2008.

[24] D. W. Hutmacher, M. Sittinger, and M. V. Risbud, "Scaffoldbased tissue engineering: rationale for computer-aided design and solid free-form fabrication systems," Trends in Biotechnology, vol. 22, no. 7, pp. 354-362, 2004.

[25] C. -K. Perng, Y. -J. Wang, C. -H. Tsi, and H. Ma, "In vivo angiogenesis effect of porous collagen scaffold with hyaluronic acid oligosaccharides," Journal of Surgical Research, vol. 168, no. 1, pp. 9-15, 2011.

[26] C. K. Chiang, M. F. Chowdhury, R. K. Iyer, W. L. Stanford, and M. Radisic, "Engineering surfaces for site-specific vascular differentiation of mouse embryonic stem cells," Acta Biomaterialia, vol. 6, no. 6, pp. 1904-1916, 2010.

[27] M. K. Smith, M. C. Peters, T. P. Richardson, J. C. Garbern, and D. J. Mooney, "Locally enhanced angiogenesis promotes 
transplanted cell survival," Tissue Engineering, vol. 10, no. 1-2, pp. 63-71, 2004.

[28] D. J. Tilkorn, A. Bedogni, E. Keramidaris et al., "Implanted myoblast survival is dependent on the degree of vascularization in a novel delayed implantation/prevascularization tissue engineering model," Tissue Engineering —Part A, vol. 16, no. 1, pp. 165-178, 2010.

[29] R. Perrot, R. Berges, A. Bocquet, and J. Eyer, "Review of the multiple aspects of neurofilament functions, and their possible contribution to neurodegeneration," Molecular Neurobiology, vol. 38, no. 1, pp. 27-65, 2008.

[30] T. A. Partridge, J. E. Morgan, G. R. Coulton, E. P. Hoffman, and L. M. Kunkel, "Conversion of mdx myofibres from dystrophin-negative to -positive by injection of normal myoblasts," Nature, vol. 337, no. 6203, pp. 176-179, 1989.

[31] G. Q. Wallace, K. A. Lapidos, J. S. Kenik, and E. M. McNally, "Long-term survival of transplanted stem cells in immunocompetent mice with muscular dystrophy," American Journal of Pathology, vol. 173, no. 3, pp. 792-802, 2008.

[32] Z. Liu, Y. Wu, and B. G. Chen, "Myoblast therapy: from bench to bedside," Cell Transplantation, vol. 15, no. 6, pp. 455-462, 2006.

[33] Y. Matsuoka and A. Inoue, "Controlled differentiation of myoblast cells into fast and slow muscle fibers," Cell and Tissue Research, vol. 332, no. 1, pp. 123-132, 2008.

[34] Y. Li and J. Huard, "Differentiation of muscle-derived cells into myofibroblasts in injured skeletal muscle," American Journal of Pathology, vol. 161, no. 3, pp. 895-907, 2002.

[35] Y. Li, W. Foster, B. M. Deasy et al., "Transforming growth factor- $\beta 1$ induces the differentiation of myogenic cells into fibrotic cells in injured skeletal muscle: a key event in muscle fibrogenesis," American Journal of Pathology, vol. 164, no. 3, pp. 1007-1019, 2004.

[36] W. Wang, H. Pan, K. Murray, B. S. Jefferson, and Y. Li, "Matrix metalloproteinase-1 promotes muscle cell migration and differentiation," American Journal of Pathology, vol. 174, no. 2, pp. 541-549, 2009. 

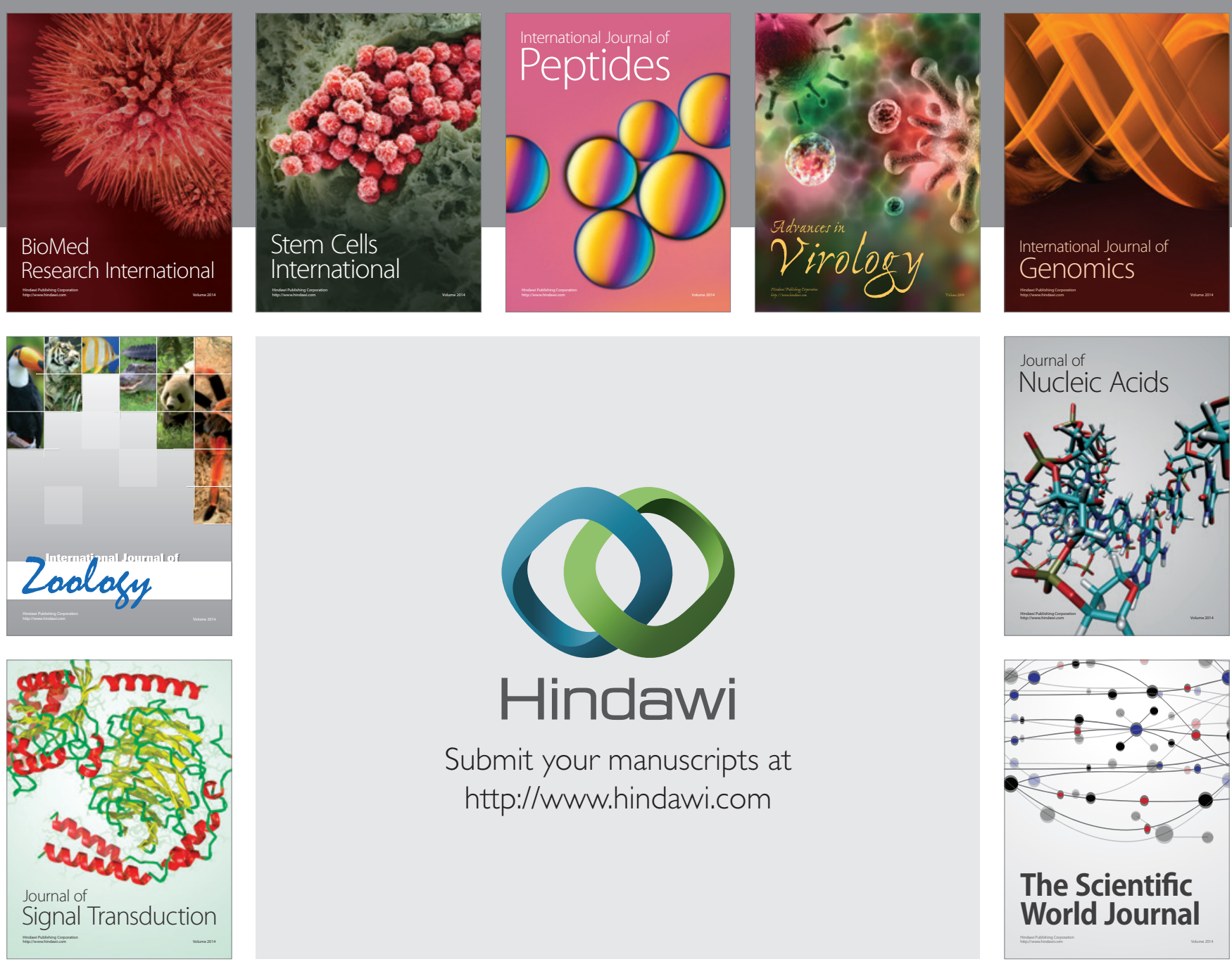

Submit your manuscripts at

http://www.hindawi.com
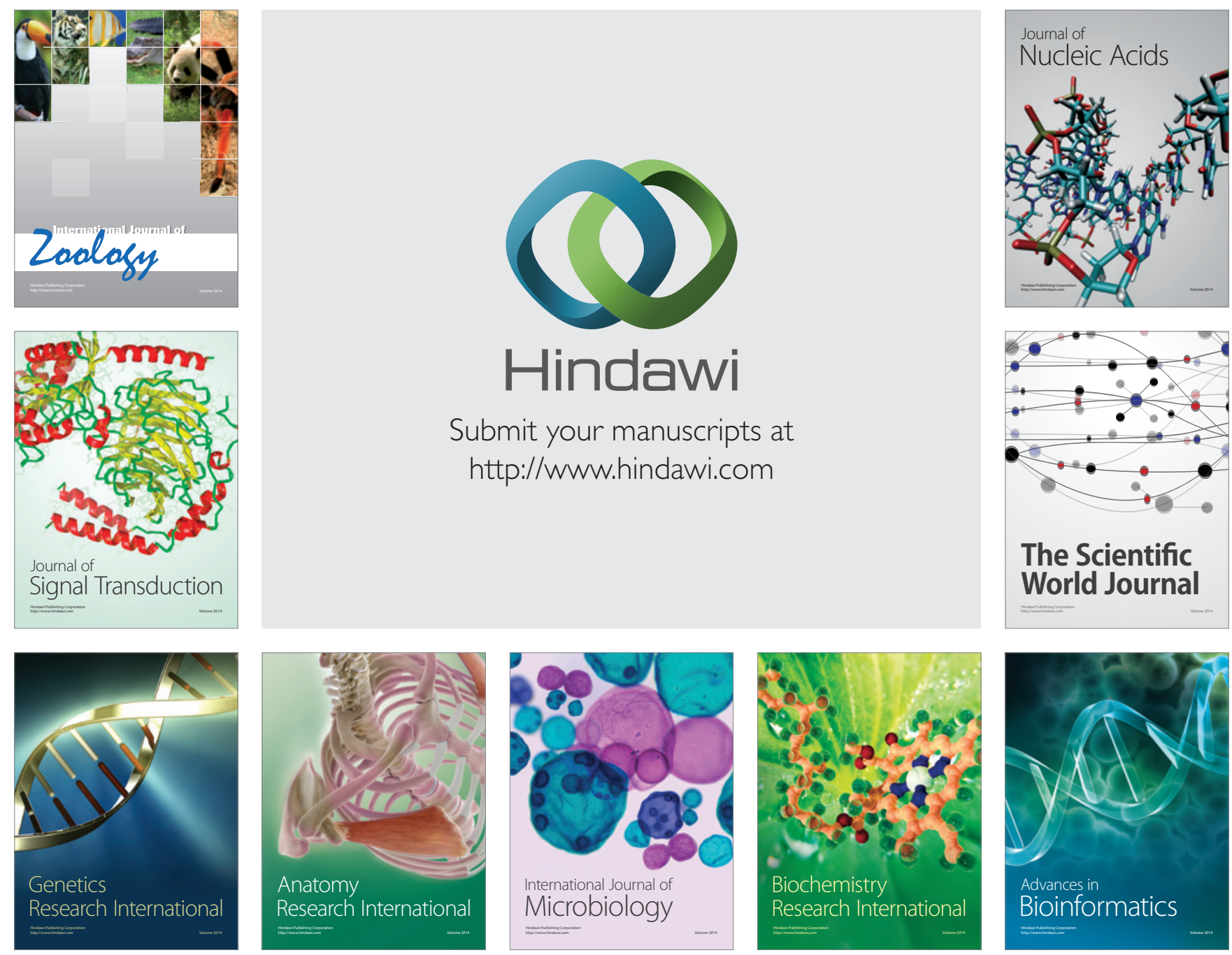

The Scientific World Journal
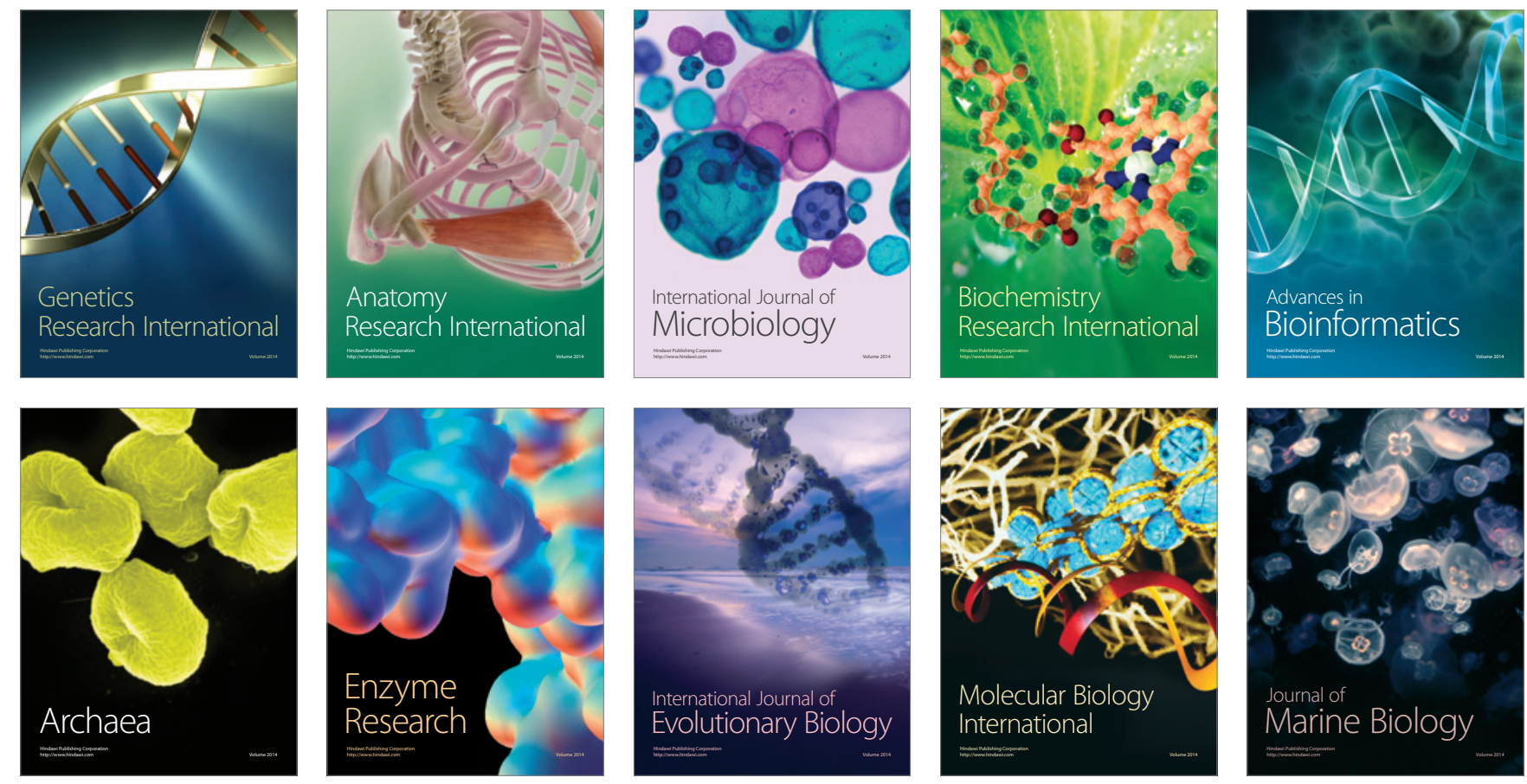